\title{
Adherence to exercise in cancer survivors
}

\section{Ashley Kornblum}

\author{
York University
}

For cancer survivors living across Canada, some of life's biggest challenges begin after treatment ends. For some people, the disease or side effects of treatment can induce physical change. For others, the cancer experience can lead to a shift in priorities, bring new insight or act as an impetus to making lifestyle changes.

Many of the negative side effects associated with the clinical manifestations of cancer can be ameliorated through medical procedures. More recently, emphasis has been placed on both behavioural and lifestyle changes that require patients to take active roles in their own health and wellness ${ }^{1}$. The role of physical activity and exercise during cancer treatment and survivorship is becoming increasingly relevant ${ }^{2}$.

A major concern for cancer survivors is their perceived sense of control. Upon diagnosis of cancer, many aspects of control, such as autonomy, are taken away. Physical activity allows people to take some control back. Furthermore, it is inversely related to all-cause mortality and has been linked with protection against several types of cancers ${ }^{2}$. Studies investigating the effects of exercise in breast and colon cancer patients have shown that a greater level of physical activity after treatment is associated with lower likelihood of disease recurrence ${ }^{3}$, reduced treatment side effects, fewer secondary comorbidities and improved quality of life ${ }^{4-7}$. Not surprisingly, discontinuation of exercise is associated with a reduction of these benefits and a return of negative symptoms ${ }^{8}$. Therefore, it is essential to ensure that cancer survivors clearly understand the importance of regular exercise and maintenance of a healthy weight over the long term ${ }^{8}$.

As the benefits of exercise appear to counteract some of the detrimental side effects of cancer treatment, these benefits can only be maintained if a consistent exercise routine is adhered to. Adherence to exercise is the extent to which individuals' exercise behaviours correspond with an exercise prescription ${ }^{8}$. For researchers implementing exercise programs, it remains one of the most complex problems to address in healthy populations and even more so in a population ailed with chronic diseases. In a cancer population, difficulty adhering to an exercise program may be related to the cancer illness itself, potential short- and long-term effects of the treatment, time elapsed following active treatment and co-morbid conditions, in addition to a myriad of factors not specifically related to cancer that can influence the exercise behaviours of people living with cancer ${ }^{3-5}$.

There is a body of literature focusing on the determinants of physical activity using a theoretical framework, which has been used to help better understand the behaviour changes and exercise patterns of cancer patients. The theory of planned behaviour has been applied in attempt to understand exercise adherence in cancer survivors and the results are very modest ${ }^{2-5,9}$. A major finding is that the strongest determinant is intention ${ }^{2-5,9}$. However, people have a tendency to over simplify their actions, as there are many issues and behaviours that are more complex and not easily predicted or measured by simply fitting them into theoretical models.

Understanding the knowledge, attitudes, behavioural and social skills associated with adhering to an exercise program is essential. However, there is a limited understanding in this area of research due to adherence measurement issues. A recent systematic review by Spence et al. emphasizes this problem with varying ways of defining adherence ${ }^{10}$. Despite advances in exercise interventions in cancer populations, there has not been accompanying advances in the standardization of the measurements of physical activity and adherence. 
Measures of self-report and observed attendance logs are often used to assess adherence, but the method of selfreport may involve possible over-estimates, or in some cases, under-estimates of physical activity and adherence. It may be associated with social desirability type responding, where participants tend to respond in a way that is viewed favourably by others or the researcher ${ }^{11}$. Consequently, self-reporting may not be the strongest methodology for understanding exercise adherence. A scientific consensus needs to be created regarding optimal adherence measurement so that specific hypotheses about how to increase exercise adherence can be developed, enabling long-term benefits of increased activity levels.

Currently, there is a limited understanding in exercise interventions about how to positively influence the longterm maintenance of healthy activity patterns, and to evaluate the impact of the relevant behaviour changes on long-term outcomes and benefits. While it is clear is that the effectiveness of exercise interventions largely depend on catalyzing motivation and adherence of the participant, this is an area to focus on as the improvements gained are valuable.

\section{References}

1. Turton, P., Cooke, H. (2000). Meeting the needs of people with cancer for support and self-management. Complementary Therapy Nurs Midwifery, 6(3):130-137.

2. Pinto, B.M. \& Hartman, S.J. (2010). Exercise and Cancer. In Jimmie C. Holland (eds.), Psycho-oncology (p. 28). New York, New York: Oxford University Press, Inc.

3. Courneya, K., Katzmaryk, P. \& Bacon, E. (2008). Physical Activity and Obesity in Canadian Cancer Survivors : Population-based Estimates From the 2005 Canadian Community Health Survey. Cancer,112(11): 2475-82.

4. Courneya, K., Mackey, J. \& Jones, L. (2000). Coping with cancer: can exercise help? Physician Sport Med, 28(5): 49-73.

5. Courneya, K., Friedenreich, C. (1999). Physical exercise and quality of life following cancer diagnosis: a literature review. Ann Beh Med, 21: 171-179.

6. Mock, V., Dow, K.H., Meares, C. et al. (1999). Effects of exercise on fatigue, physical functioning, and emotional distress during radiation therapy for breast cancer. Oncol Nurs Forum, 24: 991-1000.

7. Schwartz, A.L. (1999). Fatigue mediates the effects of exercise on quality of life. Qual Life Res, 8, 529-538.

8. Schmitz, H.H., Holtzman, J., Courneya, K.S., Masse, L.C., Duval, S., Kane, R. (2005).Controlled physical activity trials in cancer survivors: A systematic review and meta-analysis. Cancer Epidemiology Biomarkers Prevention,14(7),1588-1595.
9. Blanchard, C.M., Denniston, M.M., Baker, F., Ainsworth, S.R., Courneya, K.S., Hann, D.M., et al. (2003). Do adults change their lifestyle behaviours after a cancer diagnosis? American Journal of Health Behaviour,27 (3), 246-256.

10. Spence, R.R., Heesh, K.C., Brown, W. (2010). Exercise and cancer rehabilitation: A systematic review. Cancer treatment reviews, 36, 185-194.

11. Adams, S., Matthews, C., Ebbeling, C. et al. (2005). The effect of social desirability and social approval on selfreports of physical activity. American Journal of Epidemiology, 161(4), 389-398.

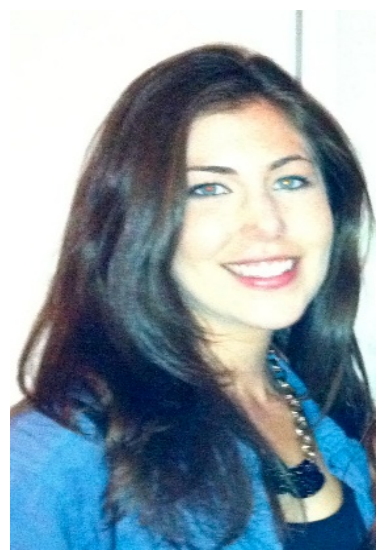

\section{Author Profile}

Ashley is currently pursuing a Master of Science under the supervision of Dr. Paul Ritvo at York University. She is also a research analyst at ELLICSR Health, Wellness \& Cancer Survivorship Centre at Toronto General Hospital. Previously, she had completed a BSc degree from McGill University. Her main research interests are in health and behaviour modification in cancer patients, focusing on patients with prostate cancer being treated with androgen deprivation therapy. 